\title{
Synthesis and Properties of Cerium Oxide/Silica Nano-composites via a Template-based Solvothermal Method
}

\author{
Liu Junliang*, Zhang Xingkai, Chen Xiulei, Qian Wei, Yan Leiming, Zhang Ming
}

Key Laboratory of Environmental Materials and Engineering of Jiangsu Province,

School of Chemistry and Chemical Engineering,

Yangzhou University, Yangzhou 225002, P.R.China

*Corresponding Author: liujunliang@yzu.edu.cn

\begin{abstract}
3-D net structured cerium oxide/silica nano-composites have been successfully fabricated via a template-based solvothermal method. Cerium oxide nano particles with sizes ranging from 50 to $60 \mathrm{~nm}$ have been homogenously adhered to the bead-bunch chain structures of silica templates with their chain's width ranging from 20 to $30 \mathrm{~nm}$. The nano-composites approached similar catalyzing effects as pure cerium oxide nano powders on the thermal decomposition of carbon black, but would improve their dispersibilities and phase compatibilities with dispersing matrix.
\end{abstract}

Keywords: Nano-composite; Rare earth oxide; Template-based solvothermal route; Thermal catalyzing properties

\section{Introduction}

Nano-composite is one of the most promising materials due to its comprehensive excellent performances, which provides an effective way to overcome the shortcomings or endow novel properties of single phase materials ${ }^{[1-2]}$. Nowadays, rare earth oxides such as cerium oxide and gadolinium oxide have attracted increasing attention because of their excellent properties especially catalyzing, electric and magnetic properties. It is believed that they can be used as novel functional fillings to supply matrixes with functional properties such as fine electrorheological effects, excellent dynamic mechanic properties and optical emitting, etc. ${ }^{[3-4]}$. However, there are still many problems when it comes to practical application, for example, rare earth oxides tend to agglomerate into island-structures for their poor compatibilities with dispersing phase, which would great deteriorate the performances of final product ${ }^{[5-6]}$.

How to effectively utilize rare earth oxides and avoid their shortcomings is one of the key problems in such fields. To overcome the problem, nano-compositing technology is an available way, therefore, we present a template-based solvothermal method to construct ceria/silica nano-composite and discuss their thermal-catalyzing properties in this paper.

\section{Experimentes}

The silica templates were fabricated via a modified hydrolysis route based on conventional Stober method: the mixture of water $(28.00 \mathrm{~mL})$ and ammonium hydroxide $(4.20 \mathrm{~mL})$ was dropped into the solution of $4.00 \mathrm{~mL}$ tetraethylortho silicate (TEOS) dissolving in $140.0 \mathrm{~mL}$ ethanol slowly at room temperature in a $250-\mathrm{mL}$ Erlenmeyer flask with continuous stirring. After stirred for another 24 hours at $50^{\circ} \mathrm{C}$ for complete reaction, the product silica templates were collected by centrifugation and vacuum-drying. The ceria/silica oxide nano-composite was synthesized with a solvothermal route: $200 \mathrm{mg}$ of the dried silica templates together with $5.00 \mathrm{~mL}$ cerium nitrate solution $(1.0 \mathrm{~mol} / \mathrm{L})$ were ultrasonically dispersed in $70 \mathrm{~mL}$ ethyl ethylene glycol and the resulting suspension was transferred into a Teflon-lined autoclave of $100 \mathrm{ml}$ capacity, 
which was then sealed and maintained at $130^{\circ} \mathrm{C}$ for 12 hours in an oil bath with magnetic stirring. After that, the obtained suspensions were cooled down to room temperature naturally and the precipitates were collected by centrifugation and washed with deionized water and ethanol for three times to eliminate the residual solvent. Subsequently, yellowish synthesized powders were obtained after vacuum drying at $80^{\circ} \mathrm{C}$. As a comparison, ceria nano-crystals were prepared by the similar solvothermal process without silica oxide templates. The phase identification of the synthesized nano-composite was performed with X-ray diffractometer (Bruker, D8 Super Speed) with $\mathrm{CuK} \alpha$ radiation and the morphologies and particle sizes of both silica templates and the as-synthesized nano-composite were analyzed by using TEM (JEOL, JEM-2100). All the samples for TEM examinations were prepared by deposition of an ultrasonically dispersed suspension of the product powders in ethanol on a carbon-coated copper grid.

\section{Resules and discussion}

Fig.1 gave the typical XRD pattern of the as-synthesized nano-composite with those of cerium oxide nano powders and silica templates for comparison. The XRD pattern of the obtained silica templates demonstrated an obvious amorphous broad peak, which indicated the silica existed in the non-crystalline state. As to the as-synthesized nano-composite, all the diffraction peaks were completely ascribed to flurite-type cerium oxide except an amorphous broad peak was from the scattering of silica templates. The co-existence of both cerium oxide's diffraction peaks and amorphous silica broad peak demonstrated the formation of the composite structures between silica templates and cerium oxide nano powders.

As shown in Fig.2, 3-D net-structured nano silica templates were successfully synthesized based on the modified Stober method and they were characterized of complex chain structures with their chains' width ranging from 20 to $30 \mathrm{~nm}$. However, there were still some bead-shape structures lying in the whole chain. The formation of such structures mainly relied on the hydrolyzing rate of TEOS. As the hydrolyzing agent, ammonium hydroxide, was dropped into TEOS slowly, TEOS hydrolyzed at low concentration ammonium hydroxide, they can not satisfy the requirement of complete hydrolyzing reaction, and therefore, they formed consecutive bead-bunch structures.

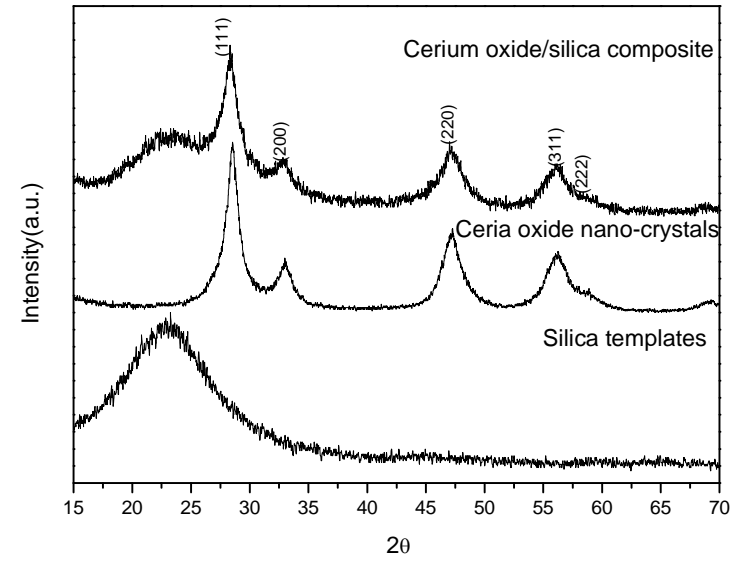

Fig.1. Typical XRD patterns of as-synthesized nano-composite with those of cerium oxide nano powders and silica templates below for comparison.
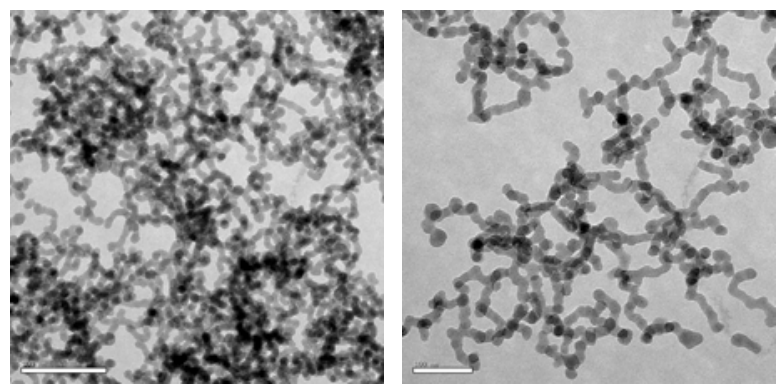

Fig.2. TEM images of silica template synthesized by hydrolysis route.

With the 3-D net-structured nano silica as the templates, cerium oxide/silica nano-composites were fabricated by solvothermal treatment as shown in Fig.3. Obviously, there were quite a lot of cerium oxide nano particles seated on the chains of silica templates. The cerium oxide nano particles were homogenously dispersed with their particles size of 50 to $60 \mathrm{~nm}$. This uniform structure may effectively improve the dispersibilities and compatibilities of cerium oxide in the matrix or dispersing phase.
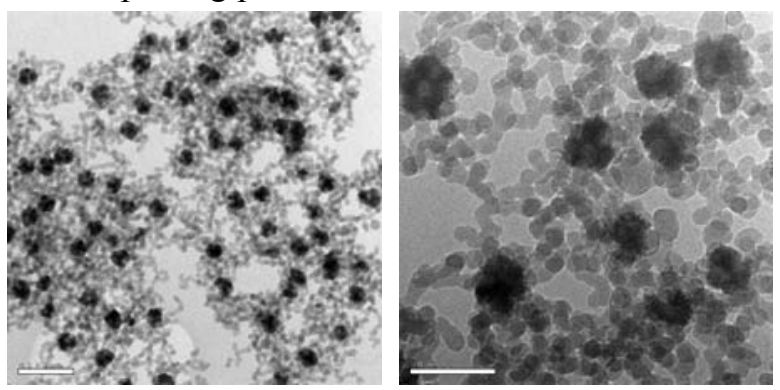

Fig.3. TEM images of the as-synthesized ceria/silicate oxide nano-composites. 
Referring to the thermal catalyzing properties of the nano-composites, carbon black was chosen to mix with the obtained nano-composites. Their TG curves were shown in Fig.4. The decomposition of carbon black was activated after it was mixed with pure cerium oxide nano powders and as-fabricated cerium oxide/silica nano-composites. Its decomposition temperature decreased from about $650^{\circ} \mathrm{C}$ to $400^{\circ} \mathrm{C}$, which demonstrated fine catalyzing abilities of carbonizing products of organic compounds. This property mainly ascribed to nano-structured cerium oxide ${ }^{[7]}$. However, the construction of the nano-composites by using amorphous silica templates did not deteriorate the catalyzing performances of cerium oxide nano powders and improved their dispersibilities and thermal stabilities to some extend. Carbon black was incomplete decomposition product of organic components, which would decrease the performances of the whole devices, for example, the pollution from automobile exhaust ${ }^{[7]}$ or the carbon on the electrode of SOFC hindered the transmission of carriers ${ }^{[8]}$. The as-fabricated nano-composite provided an available way to solve these problems.

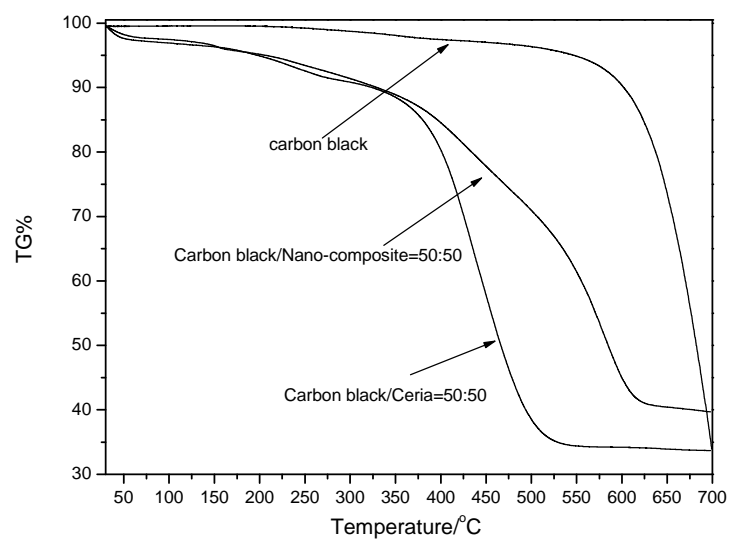

Fig.4. TG curves of the obtained nano-composites mixed with carbon black

\section{Conclusions}

In summary, the amorphous silica with 3-D net structure has been successfully fabricated by modified Stober method. They were characterized by bead-bunch chain with chain's width of 20 to $30 \mathrm{~nm}$. With using it as the template, cerium oxide particles with sizes of 50 to $60 \mathrm{~nm}$ have been attached to the chains of the silica template and formed uniform nano-composite structure. The as-fabricated nano-composites maintained the catalyzing ability similar to pure cerium oxide nano powders but provided a valid way to improve their compatibilities with dispersing matrix.

\section{Acknowledge}

The authors gratefully acknowledge the financial supports from National Natural Science Foundation of China (No.51103125 and No. 51273172), the Higher Education Science Foundation of Jiangsu Province (No.10KJB430018), the Project Funded by the Priority Academic Program Development of Jiangsu Higher Education Institutions and the Graduate Student Innovation foundation of Jiangsu Province, P. R. China (No.CXLX11_1003).

\section{References}

(1) Hailin Cong, Maciej Radosz, Brian Francis Towler, Youqing Shen, Separa. Purif. Tech. 55,281-291,2007.

(2) Jian Liu, Shizhang Qiao, Qiuhong Hu, Gaoqing Lu, Small, 4, 425-443,2011.

(3) Seung Soo Lee, Huiguang Zhu, Elizabeth Q.Contreras, Arjun Praksh, Hema L. Pupplala, Vicki L. Colvin, Chem. Mater. 24,424-432, 2012.

(4) Yangfeng Huang, Yebin Cai, Dongkai Qiao, Hao Liu, Particuology, 9,170-173,2011.

(5) Shuxue Zhou, Yijing Yin, Bo You, Limin Wu, Min Chen, Macromol. Chem. Phys. 208,2677-2685,2007.

(6) Feliks Bezati, Valérie Massardier, Jean Balcaen, Daniel Froelich, Polym. Degrad. Stabi. 96,51-59,2011.

(7) Nicholas C. Strandwitz, Galen D. Stucky, Chem. Mater. 21,4557-4582,2009.

(8) Park S.D., Vohs J.M., Gorte R.J., Nature, 404,265-267,2000. 